\title{
Features of multi-particle processes on light nuclei in target experiments
}

\author{
B.A. Iskakov ${ }^{1}$, A. Kh. Argynova, A.D. Beisenova, T.N. Kvochkina, A.A. Loctionov, \\ Y.M. Tautayev, T.Kh. Sadykov \\ Satbayev University, Institute of Physics and Technology \\ Almaty, Kazakhstan \\ E-mail: leodel@mail.ru
}

\begin{abstract}
The system size problems are the important component of the multi-particle dynamics, which, however, is not sufficiently taken into account in the cosmic ray researches. Therefore, in addition to a broad and important program of new collider and target experiments to study the interactions of protons with light nuclei, we propose to consider the features of multi-particle processes on light nuclei. In the work these problems are studied in the centrality selected interactions of light $-(\mathrm{C}, \mathrm{O}, \mathrm{Ne})-($ beam $)$ and heavy $-(\mathrm{Au}, \mathrm{Pb})-($ beam $)$ ions with light $(\mathrm{C} / \mathrm{N} / \mathrm{O})-($ target $)$ and heavy $-(\mathrm{Ag} / \mathrm{Br})-($ target $)$ nuclei on the data of JINR-AGS-SPS target emulsion experiments with limited statistics. In order to reveal the possible system structure dependence, the analyses of E-by-E fluctuations on the longitudinal event shape and multiplicity are performed in two directions: on vertically, - for different (beam-target) pairs of nuclei under certain impact parameters, and on horizontally, - for the same (beam-target) pairs of nuclei on different impact parameters. The strong fluctuation enhancement for the central collisions of $(\mathrm{C}$, $\mathrm{O}, \mathrm{Ne})-$ beam $+(\mathrm{C} / \mathrm{N} / \mathrm{O})-$ target was interpreted as the sign of intrinsic virtual alpha-clustering in light nuclei.
\end{abstract}

36th International Cosmic Ray Conference -ICRC2019-

July 24th - August 1st, 2019

Madison, WI, U.S.A.

${ }^{1}$ Speaker 


\section{Introduction}

Improving the understanding of the dependence of multiple processes on the size and structure of the interaction region is one of the most pressing problems in high energy physics [1]. At high energies, the study of the interactions of "small" ( $\left.p+p, p / d /{ }^{3} \mathrm{He}+\mathrm{A}\right)$ and "large" $(\mathrm{A}+\mathrm{A})$ systems in the RHIC and LHC experimental systems showed $[2,3]$ that the structures of two and/or multi-particle correlations in the azimuthal distribution of secondary particles are of a similar nature. The analysis first of all led to the conclusion $[4,5]$ about the need to revise the concepts in which the properties and properties of dense material objects are studied, as a result of which changes in nuclear substances are detected, mainly as a reference point when comparing data [1].

NA61/SHINE with a fixed target on the SPS [6] as part of a systematic search for the critical behavior of strongly interacting matter. The possibilities of protons, light, medium, and open nuclei $(\mathrm{Be}, \mathrm{Ar}, \mathrm{Xe}, \mathrm{Pb})$ in the full range of SPS energies were studied in order to identify possible nonmonotonic behavior in fluctuations of the parameters of multiple processes. However, the analysis of the interaction in $\mathrm{p}+\mathrm{p}, \mathrm{Be}+\mathrm{Be}$, and $\mathrm{Ar}+\mathrm{Sc}$ collisions has not yet provided any indications of this behavior [6]. It is necessary to study the problems associated with a change in the properties of a substance under conditions of low energy and high density of baryonic acid.

In the near future, such new experiments will be implemented at JINR in the NICA project [7], in the GSI center in the FAIR project [8] and in the J-PARC project in Japan. For this purpose, the STAR RHIC experiment is preparing a new energy scanning program (BES II) and work with fixed targets [9].

In this context, the problems of the formation of new approaches to the analysis of the dependence of multiple processes on the size and structure of the initial interaction region are very important. The results of such work carried out both in the stratospheric experiment in cosmic rays at high energies and in accelerator experiments at JINR-AGS-SPS energies are presented in this article. The second section compares the multiple processes in the interactions of protons and light nuclei of cosmic rays. A detailed comparison of the multiple processes in the central interactions of light, medium, and heavy nuclei in targeted emulsion experiments is carried out in the third section.

\section{Comparison of multiple processes in the interactions of protons and light nuclei of cosmic rays}

In cosmic rays, the work was performed $[11,12]$ according to the data of the LPI-FTI "Stratosphere" collaboration at $\mathrm{TeV}$ energies. Computer intermittence analysis of collisions of protons and primary light nuclei with targets in an X-Ray emulsion chamber showed that there is a significant difference in the "true" dynamic fluctuations of charged particles for these events. Threefold averaging of factorial moments in the modified method of "vertical intermittensity analysis" developed by our group - by events, by the starting point of the analysis interval and by the size of the interval $\angle \mathrm{F}_{\mathrm{q}}>$ allowed us to overcome the empty bin effect effect and obtain estimates of the exponent $\varphi(q)$ of the intermittency for moments of high 
rank $\mathrm{q}=8$. This is two units higher than the standard approach, which is very important, since it is the many-particle fluctuations that are the most informative. Qualitatively, the results of the calculations showed a well-known trend toward a decrease in the intermittency indices during the transition from $r$ to AA collisions. Quantitatively, two-dimensional rafts $\varphi(q)$ at high $q$ ranks clearly showed two different q-behavior areas for events formed by protons and light nuclei.

An additional analysis of the spectra of transverse momenta of all gamma rays in the soft region (up to $2 \mathrm{GeV} / \mathrm{c}$ ) again demonstrated a large (exceeding statistical error) difference between proton and nuclear interactions.

It should be emphasized that the results of the Stratosphere experiment on the dependence of multiple processes on the size of the interaction region are based on an analysis of the spectra of transverse momenta and pseudo-rapidities for all particles in each event. At comparable energies, such a result is very difficult to achieve at colliders, where the production of only individual particles of the event, moving forward at small angles, is studied in detail. Therefore, a direct comparison of our data on the Stratosphere experiment with collider data is currently difficult due to the inclusive nature of the LHCf data inclusiveness [13].

\section{Comparison of multiple processes in the central interactions of light, medium and heavy nuclei at JINR-AGS-SPS energies}

A detailed analysis of multiple processes was performed using data from the international EMU collaboration [13]. Comparison of multiple processes was carried out in the central interactions of light - $(\mathrm{C}, \mathrm{O}, \mathrm{Ne})$, medium - $(\mathrm{Si}, \mathrm{S})$ and heavy - $(\mathrm{Au}, \mathrm{Pb})$ ions with heavy $(\mathrm{Ag} / \mathrm{Br})$ and light $-(\mathrm{C} / \mathrm{N} / \mathrm{O})$ nuclei in targeted emulsion experiments. The identical capabilities of high-precision measurements of both storm particles and fast fragments - spectators open the way to an effective solution of the problem posed. Experiments at energies of $3.7 \mathrm{~A} \mathrm{GeV}$ for light - $(\mathrm{C}, \mathrm{O}, \mathrm{Ne})$ and medium - $(\mathrm{Si}, \mathrm{S})$ nuclei were performed at the JINR, at energies of $14 \mathrm{~A}$ $\mathrm{GeV}$ for $\mathrm{Si}$ ions, and at energies of $10.7 \mathrm{~A} \mathrm{GeV}$ for $\mathrm{Au}$ ions - Brookhaven, at energies of $200 \mathrm{~A}$ $\mathrm{GeV}$ for $\mathrm{S}$ ions and at energies of $158 \mathrm{~A} \mathrm{GeV}$ for $\mathrm{Pb}$ ions, at $\mathrm{CERN}$.

In order to study the processes of multiparticle dynamics, only events with a large multiplicity $\mathrm{N}_{\mathrm{s}}>1 / 3\left(\mathrm{~N}_{\mathrm{s}}\right)_{\max }$ were selected for analysis, where $\left(\mathrm{N}_{\mathrm{s}}\right)_{\max }$ is the maximum multiplicity in the corresponding experiment. The dependence of the processes on the size of the interaction region has been studied in collisions [14-15] of "light-lungs" - $(\mathrm{C}, \mathrm{O}, \mathrm{Ne})+(\mathrm{C} / \mathrm{N} / \mathrm{O})$ and "medium-light" - $(\mathrm{Si}, \mathrm{S})+(\mathrm{C} / \mathrm{N} / \mathrm{O})$ and "heavy-heavy" - $(\mathrm{Au}, \mathrm{Pb})+(\mathrm{Ag} / \mathrm{Br})$ nuclei.

\section{Analysis of the distribution of multiplicity in the central collisions of light, medium and heavy nuclei}

The qualitative nature of the increase in fluctuations of multiplicity with a decrease in the size of the interaction region was already established in the analysis of multiple fragmentation correlations. For a quantitative study of the plurality distributions, we must eliminate, or at least minimize, uncontrolled fluctuations in the volume of interaction. In each individual collision, the nucleons of the incident nucleus participating in the interaction are adequately determined in our algorithm for estimating the degree of centrality. In order to exclude fluctuations in the target nucleus, only the most central nuclear collisions should be selected [16]. Comparative 
analysis of fluctuations in the relative multiplicity of $\mathrm{N}_{\mathrm{s}} / \mathrm{N}_{\text {smax }}$ in the most central lung-lungs $(\mathrm{C}, \mathrm{O}, \mathrm{Ne})+(\mathrm{C} / \mathrm{N} / \mathrm{O})$, medium-light $-(\mathrm{Si}, \mathrm{S})+(\mathrm{C} / \mathrm{N} / \mathrm{O})$ and heavy -heavy $-(\mathrm{Au}, \mathrm{Pb})+(\mathrm{Ag} / \mathrm{Br})$ nuclear collisions showed that the widths in each type of collisions are inversely proportional to the volume of interacting systems. The largest fluctuations in multiplicity were recorded in light-light collisions of nuclei $-(\mathrm{C}, \mathrm{O}, \mathrm{Ne})+(\mathrm{C} / \mathrm{N} / \mathrm{O})$. The results are presented in Figure 1.
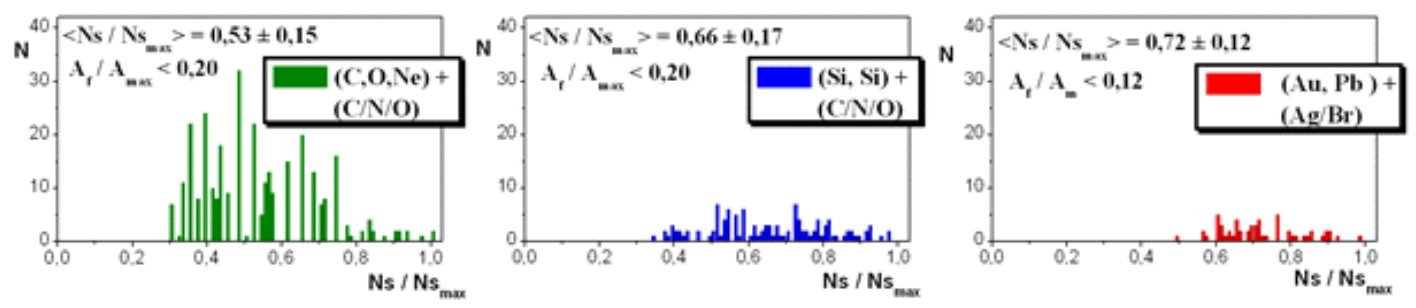

Figure 2 - Distributions of the relative $\mathrm{N}_{\mathrm{s}} / \mathrm{N}_{\text {smax }}$ multiplicity in the central interactions of the lungs - $(\mathrm{C}, \mathrm{O}, \mathrm{Ne})$, medium - $(\mathrm{Si}, \mathrm{S})$ and heavy - $(\mathrm{Au}, \mathrm{Pb})$ ions with heavy $-(\mathrm{Ag} / \mathrm{Br})$ and light $(\mathrm{C} / \mathrm{N} / \mathrm{O})$ cores

The combination of these results forms a very important trend. On the one hand, it does not fit into the standard behavior for various measures [17-18] of statistical fluctuations. On the other hand, such a result can provide a new look at the presence of cluster geometry in collisions of light nuclei. These results were interpreted [17-18] as a clear signal of manifestation of the internal alpha-clustering of light nuclei in multiple processes at JINR-AGS-SPS energies.

In this context, the recent results of mathematical modeling of asymmetric interactions of light-heavy nuclei should be noted, where it was shown [19] that the cluster structure of an excited fireball can be formed, reflecting the alpha-cluster nature of the incident nucleus.

Table 1

\begin{tabular}{|c|c|c|}
\hline Type of interaction of nuclei & $\mathrm{A}_{\mathrm{f}} / \mathrm{A}_{\max }$ & $<N s / N s_{\max }>$ \\
\hline Central collisions $\mathrm{C}, \mathrm{O}, \mathrm{Ne}+(\mathrm{C} / \mathrm{N} / \mathrm{O})$ & 0.20 & $0.53 \pm 0.15$ \\
\hline Central collisions $\mathrm{Si}, \mathrm{S}+(\mathrm{C} / \mathrm{N} / \mathrm{O})$ & 0.20 & $0.66 \pm 0.17$ \\
\hline Central collisions $\mathrm{Au}, \mathrm{Pb}+(\mathrm{Ag} / \mathrm{Br})$ & 0.12 & $0.72 \pm 0.12$ \\
\hline
\end{tabular}

\section{Conclusion}

- Strong experimental signals of the dependence of multiple processes on the size of the interaction region in both space and accelerator experiments have been established.

- A sharp increase in fluctuations in multiplicity and pseudo-rapidity was revealed in the central interactions of light-light $-(\mathrm{C}, \mathrm{O}, \mathrm{Ne})+(\mathrm{C} / \mathrm{N} / \mathrm{O})$ nuclei compared to medium-light - $(\mathrm{Si}$, $\mathrm{S})+(\mathrm{C} / \mathrm{N} / \mathrm{O})$ and heavy-heavy - $(\mathrm{Au}, \mathrm{Pb})+(\mathrm{Ag} / \mathrm{Br})$ nuclear collisions. An increase in fluctuations with a decrease in the interaction region may reflect a violation of the correct spherical picture of the nucleus during the transition from heavy-heavy to light-light collisions 
of nuclei. Internal alpha clustering of light nuclei may be one of the most likely sources of this fluctuation behavior.

In addition, these data can be used in other areas of high energy physics and cosmic ray physics [20-21].

\section{References}

[1] J. Schukraft, Status and Key open Questions in Ultra-Relativistic Heavy-Ion Physics, Quark Matter 2017 is the XXVI international conference on ultrarelativistic heavy-ion

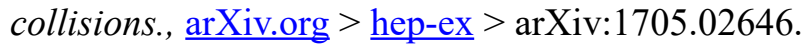

[2] A. Ohlson, Collective behaviour in small systems, - an experimental perspective, Quark Matter 2017 is the XXVI international conference on ultrarelativistic heavy-ion collisions., http://indico.cern.ch/event/433345/contributions/2321604/attachments

[3] K. Dusling, W. Li, B. Schenke, Novel Collective Phenomena in High-Energy ProtonProton and Proton-Nucleus Collisions. arXiv.org > nucl-ex > arXiv:1509.07939.

[4] B. Schenke, Origins of collectivity in small systems, Quark Matter 2017 is the XXVI international conference on ultrarelativistic heavy-ion collisions., http://indico.cern.ch/event/433345/contributions/2321605/attachments/1409943/2157580/S chenke-QM2017.pdf.

[5] W. Li, Collective flow from AA, pA to pp collisions - Toward a unified paradigm, Quark Matter 2017 is the XXVI international conference on ultrarelativistic heavy-ion collisions., arXiv.org > nucl-ex > arXiv:1704.03576

[6] A. Aduszkiewicz, Recent results from NA61/SHINE, Quark Matter 2017 is the XXVI international conference on ultrarelativistic heavy-ion collisions., http://indico.cern.ch/event/433345/contributions/2311840/attachments/1407215/2151926/ Antoni_Aduszkiewicz_QM2017_slides.pdf.

[7] V. Kekelidze, Feasibility study of heavy ion collision physics at NICA, Quark Matter 2017 is the XXVI international conference on ultrarelativistic heavy-ion collisions., http://indico.cern.ch/event/433345/contributions/2358352/attachments/1408583/2154124/ Kekelidze.pdf.

[8] H. Caines, The search for critical behavior (and other featuresof the QCD phase diagram). Status and future, Quark Matter 2017 is the XXVI international conference on ultrarelativistic heavy-ion collisions., http://indico.cern.ch/event/433345/contributions/2321607/attachments/1410551/2157257/ CainesQM17.pdf.

[9] P. Sender, The heavy-ion program at the future FAIR facility, Journal of Physics: Conference Series. - 2017. - V. 798.-N012062.

[10] https://fair-center.eu/for-users/experiments/nuclear-matter-physics/news/news.html.

[11] A.Kh. Argynova, N.I. Kochelev, T.N. Kvochkina et al., Proton and light ion interactions in cosmic ray experiment "Stratosphere" in the comparison with the recent collider results, Poster-Report on 34-th Intern. Cosm. Ray Conf., PoS (ICRC 2015).

https://pos.sissa.it/cgi-bin/reader/conf.cgi?confid=236, session-2514 
[12] A.A. Loctionov, A.Kh. Arginova, A.Sh. Gaitinov and T.N. Kvochkina, The study of initial conditions in collisions of light, intermediate and heavy nuclei, XIX International Symposium on Very High Energy Cosmic Ray Interactions, https://doi.org/10.1051/epjconf/201614514004.

[13] B.Maksiak, Two-particle correlations in $p+p$ and $P b+P b$ collisions at SPS energies, Doctoral thesis, Warsaw University of Technology, 2016.

[14] A.Kh. Argynova, A.Sh. Gaitinov, A.A. Loctionov, The analysis of multiplicityfragmentation correlations in asymmetrical nuclear collisions at JINR-AGS-SPS energies, Report on the Intern. Conf. ICNRP'13, "Nuclear and Radiation Physics".

[15] A. Kh. Argynova, A. Sh. Gaitinov, A.A. Loctionov, Strong fluctuations in initial conditions for interactions of light nuclei at high energies, report on the Intern. Conf. «Modern Problems of Physics and New Technologies», Izv. NAN RK, ser. phys.-mat. 2014, N 3, p. 92-93.

[16] V. Klochkov, I. Selyuzhenkov, Centrality determination in heavy-ion collisions with the CMB experiment, Journal of Physics: Conference Series. 2017. - V.798. - N 012059.

[17] A.Kh. Arginova, A.Sh. Gaitinov, T.N. Kvochkina, A.A. Loktionov, System size dependence of multiparticle processes, produced high-energy protons and nuclei, Report on the Intern. Conf. ICNRP'17, "Nuclear and Radiation Physics".

[18] A.Kh. Argynova, A.Sh. Gaitinov, A.A. Loctionov, The analysis of multiplicityfragmentation correlations in asymmetrical nuclear collisions at JINR-AGS-SPS energies, Report on the Intern. Conf. ICNRP'13, "Nuclear and Radiation Physics".

[19] E. Bratkovskaya, HIC for FAIR, Workshop on Fluctuations and Correlation, Frankfurt, 2015.

[20] Iskakov B.A. et al., Studies of EAS in the Tien-Shan high-mountain scientific station, Journal of Physics: Conf. Series 1337 (2019) 012004

[21] Argynova A.Kh. et al., The perspective fundamental cosmic rays physics and astrophysics investigations in the Tien Shan high-mountain scientific station, News of the National Academy of the Republic of Kazakhstan Ser. Geology and Technology Sciences V. 6, N438 p.121-138. 